\title{
Pressure and velocity conditions in home health care accessory for babies
}

\author{
Blanka Skočilasová ${ }^{1, *}$, Jan Skočilas ${ }^{2}$, and Josef Soukup ${ }^{1}$ \\ ${ }^{1}$ University J. E. Purkyně in Ústí nad Labem, Faculty of Mechanical Engineering, Institute of \\ Machinery and Energetics, Na Okraji 1001, 40096 Ústí nad Labem, Czech Republic \\ ${ }^{2}$ Czech Technical University in Prague, Faculty of Mechanical Engineering, Department of Process \\ Engineering, Technická 4, 16607 Praha 6, Czech Republic
}

\begin{abstract}
The home health care accessory for babies is selected for investigation. The objectives are to find out the maximal under-pressure and air velocity, which is possible to reach by accessory Nosko ${ }^{\circledR}$ using standard home vacuum cleaner recommended by manufacturer of the accessory. CFD software is used for prediction of the flow characteristics of the air in the body of the accessory. Also simple experiment with the trace is performed in the transparent body. The flow characteristic of the Nosko ${ }^{\circledR}$ is obtained by systematic simulation and can be used for further engineering calculation and detailed investigation. Maximum flow rate of the air through the accessory under maximal under pressure generated by home vacuum cleaner was 2.6 litres. $\mathrm{s}^{-1}$ which is comparable with results of investigation of the human breathing in the literature source.
\end{abstract}

\section{Introduction}

The progress in home health care is obvious in the every following human generation, which is reflected by utilization of the new materials and technologies. Above all the evolution of the new preparations, gadgets and supplements or accessories is the most prominent in the field of baby care. One of these accessories is the tool for removing of the phlegm from baby sinus nasal for example. We have chosen it for our investigation. The Nosko ${ }^{\circledR}$ is the product of the company Ranyak Gyyrgy, Budapest, Hungary. It is plastic draft tube extender, which is inserted into the baby nose. The second end is connected to the home vacuum cleaner. However, it seems that it is drastic or torturous tool, the manufacturer is boasting about the high efficiency of the phlegm removal and prevention to the phlegmasia of upper respiratory tract or otitis media (inflammatory diseases of the middle ear). The product passed by clinical tests and moreover, according to long time users references, the satisfaction of using this as the prevention of diseases (also for adults) prevails. We stood in front of the decision (in the role of the parents as well as engineers) to use standard methods or new surroundings recommended accessory Nosko®. Despite of all positive references, we firstly investigated and tested the accessory before first application to baby. The results of the investigations are presented in this contribution.

\footnotetext{
* Corresponding author: blanka.skocilasova@ujep.cz
} 
In the field of the fluid flow in nozzles, there are many basic and specialized literature [1-3]. This issue is deeply investigated and continuously broaden out till present. The many theoretical and experimental studies exist, focused on the pressure and velocity profiles in or around the nozzle for different purposes, flow regimes or method of the nozzle operation (discharge or suction). Today, CFD models are standard tools of the scientists, which save the time and cost of experiment, however the result of the simulation is necessary at least partially to compare with experiment or basic theory. Many author use CFD software for investigation of the fluid flow through nozzle [4], e.g. distribution of the air in the building or room for air-condition [5-6], cavitation in the fuel injection nozzles [7] or fluid flow together with heat transfer of the impinging stream on the cooled or heated wall [8]. From these articles the general rules and recommendation raised, which deals with set up of the mathematical models from proper geometry generation and simplification, CGI analysis [9], density and quality of the mesh including suitability of the different type of the mesh elements, up to recommendation for different models of turbulence and relationship between primitive variables in Navier-Stokes equations and continuity equations.

Nosko ${ }^{\circledR}$ is utilized together with dome vacuum cleaner. The European standard [10] exists for these kind of the cleaners commonly available in the market, which above all specifies the limits of operation parameters for dry or wet cleaner. Maximal reachable uder pressure is limited to $30 \mathrm{kPa}$ for dry cleaning. This standard also states the maximal volumetric flow-rate of the air in the cleaner, which is from 20 up to 40 litres. $^{-1}$ for standard vacuum cleaners.

From above mentioned information the doubt is arising. Is it ever reasonable to interconnect the Nosko ${ }^{\circledR}$ with home vacuum cleaner on the one side with baby sinus nasal on the other side? Is there any threat of baby health impediment? Several authors describe standard pressure relation in the sinus nasal of adults under normal breath condition. The flow rate of the air via the nose during maximum-effort vital capacity maneuvers reached 4.32 litres. $\mathrm{s}^{-1}$ on inspiration and 7.09 litres. $\mathrm{s}^{-1}$ on expiration period [11]. The most often these studies are published in the special medical journals, however we have doubt about the clinical tests existence, which deals with investigation of maximal under pressure of the air in the baby sinus nasal limited by baby health impediment. Therefore this article deals with investigation of the maximal available operation conditions reachable by Nosko ${ }^{\circledR}$ accessory together with home vacuum cleaner. Pertinent examination of these results from baby health point of view is conveyed to medical experts with respect.

Above all the article objective is to find out the maximal under-pressure and air velocity, which is possible to reach by Nosko ${ }^{\circledR}$ using standard home vacuum cleaner. The simulation results of air flow in accessory by CFD tool were partially compared with simple experiment considering the observation of the flow contours - streamlines of the tracer. The flow characteristic on the accessory was also obtained from simulations.

\section{Materials and methods}

For the investigation the standard home health care accessory for babies, Nosko ${ }^{\circledR}$ was chosen for this study, which is commonly available in the market. Nosko ${ }^{\circledR}$ is the product of the company Ranyak Gyyrgy, Budapest, Hungary. It is transparent plastic draft tube extender, which is inserted into the baby nose. The second end is connected to the home vacuum cleaner. The general look of this tool is presented in the Figure 1. The narrowed part is inserted into the baby nose. The sleeve which is perpendicular to the axis of the accessory is connected by silicon tube and reducer with the hose of the home vacuum cleaner. The inlet jet cavity is prolonged and its diameter is extended by inserted straight tube with constant thickness almost to the bottom end of the outer shell of the accessory body. The air enters to the Nosko ${ }^{\circledR}$ by narrow nozzle. The stream channel is gradually 
enlarged to the diameter of inserted tube. The air axially continues to the end bottom, where it is completely turning round to the counter flow direction. Then the air flows by annular section between outer shell and inserted tube and finally it leaves the body in perpendicular direction to the axis by sleeve. The phlegm is sucked in with air but it doesn't leave the Nosko ${ }^{\circledR}$ and remains in the body, because the described geometry also serves as gas-liquid separator. The body is easy demountable to three pieces for water cleaning.

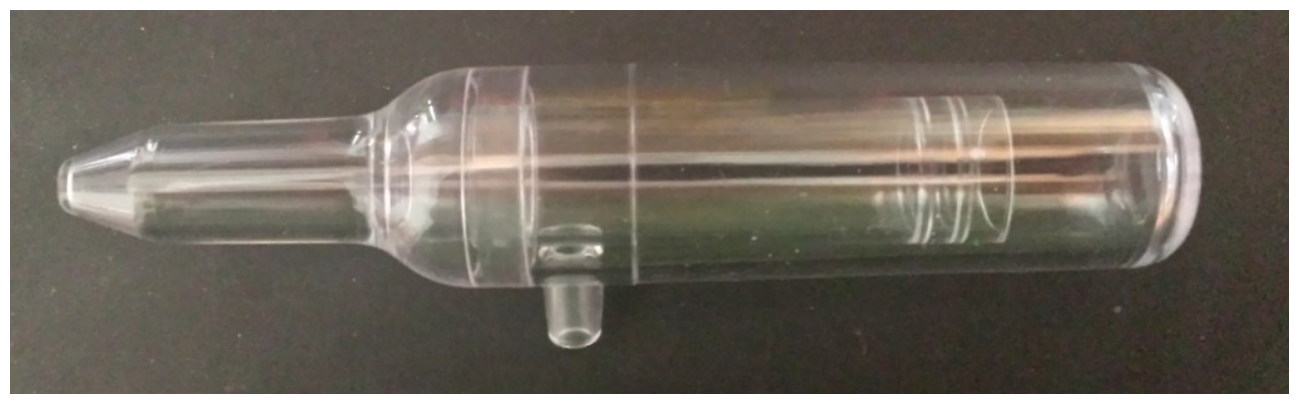

Fig. 1. Side view of the Nosko®.

The geometry for CFD simulation was generated according to the measured dimensions of the body in CAD/CAM modeller. The wall were completely remove because only the negative of the inner volume was used for simulations, see Figure 2.

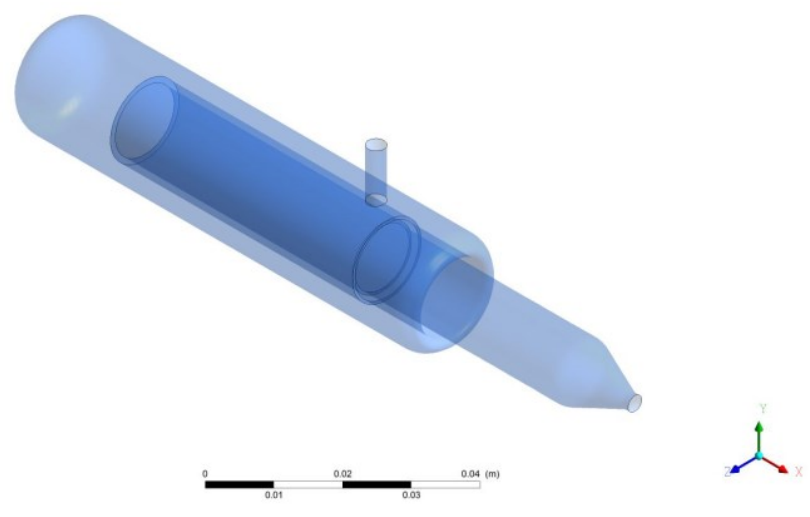

Fig. 2. Final geometry of model for CFD simulation.

For CFD simulation and mesh generation the packages of the software ANSYS ${ }^{\circledR}$ were used. The simulations were performed in Fluent ver.16.0 software. The CGI analysis [9] was also performed before systematic investigation of the flow characteristics. The final mesh has approximately 500.000 element which was acceptable for further simulation. Due to rounded geometry the tetrahedral elements prevails. Quality of the mesh was inspected (especially orthogonal quality and skewness) and the mesh was optimized replacing the worst elements and by using improving mesh quality tool in Fluent software. The gravity forces and compressibility of the air were not assumed in the calculations. The physical properties of the air was considered under standard pressure and temperature 
conditions. The boundary conditions were set up to pressure inlet and outlet respecting the maximal under pressure available for home vacuum cleaners [10]. After the maximum flowrate was obtained, the boundary conditions were changed to mass inlet and pressure outlet to investigate whole range of the flowrates and flow characteristic of the Nosko ${ }^{\circledR}$. The k- $\varepsilon$ SST model was used for turbulence regime of air flow. The solver was set upped to double precision, and second order upwind for momentum and pressure. SIMPLE scheme was used for relation between primitives' variables (velocity and pressure). At least 5000 iterations were performed at each simulation for reaching the stable solution, however the oscillation of the residuals occurred. Therefore the average value of pressure from last 500 iteration was calculated for assessing the pressure drop.

Also simple experiments were carried out to observe the air flow streams in the body of the Nosko®. The basic home aerosol/fog generator Ultrasonic Nebulizer LD-250U, Nantong Co. Ltd., China, was used for production of the fog which was sucked into the body. The flow of the tracer was recorded by camera SONY HDR-CX115, Sony Co., China against the black background. The recorder file was investigated frame by frame and the best results of the tracer streams are presented. The velocity of the incoming stream into the Nosko ${ }^{\circledR}$ connected to home vacuum cleaner Miele Compact C1 PowerLine was also measured using the AHLBORN Almemo 2590 anemometer with hot wire probe AHLBORN ALMEMO FVAD15S240, but with unsuccessful result, because the stream velocity at the inlet exceed the measuring range of the probe which was $40 \mathrm{~m} \cdot \mathrm{s}^{-1}$.

\section{Results and discussion}

The results of the simulations as well as the result of the simple experiment are presented here. The main graphical outputs of the simulation are the pressure and velocity distribution presented in the Figure 3 and 4. The investigated range of the pressure drop was from $20 \mathrm{~Pa}$ up to $56 \mathrm{kPa}$ and corresponding flow rate from 0.03 litres. $\mathrm{s}^{-1}$ up to 2.6 litres. $\mathrm{s}^{-1}$. All results presented here in the graphical form represent the flow condition 2.6 litres. $\mathrm{s}^{-1}$ and pressure drop $56 \mathrm{kPa}$ (absolute pressure $47 \mathrm{kPa}$ ).
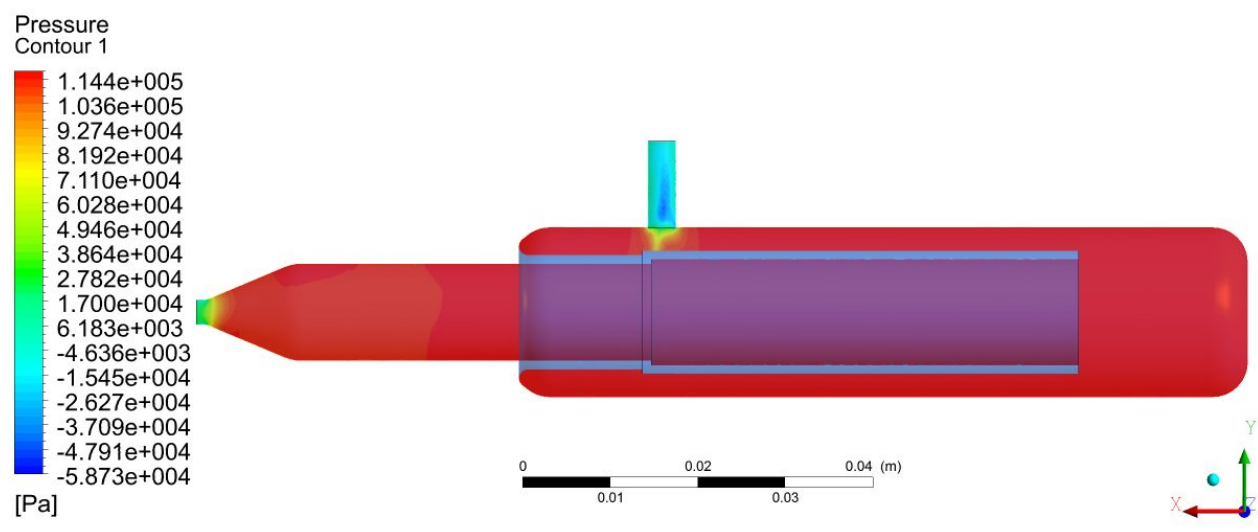

Fig. 3. Pressure contours for 2.6 litres. $\mathrm{s}^{-1}$.

The maximal local pressure drops which are the main part of the global pressure drop of the investigated body are located at the inlet and outlet to the system. There are extreme reduction and expansion of the cross-section area, see Figure 3. It seems that the bending of the main stream by turning round at the end of the outer shell together with distribution of the stream from circular to annular cross section and bending and collecting of the whole 
stream into the outlet have no significant effect on the overall pressure drop respecting the uniform colour of the pressure in the main body.
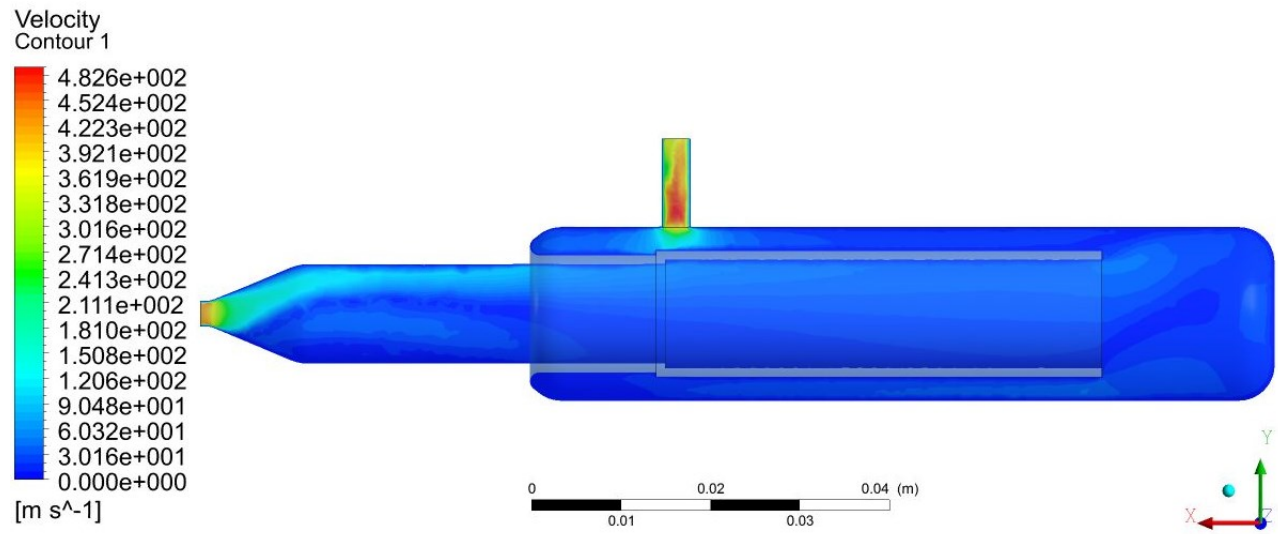

Fig. 4. Velocity contours for 2.6 litres.s ${ }^{-1}$.

In the Figure 4 and 5 the contour of the velocity profile and streamlines are presented respectively. According to asymmetry of the body geometry in the XY plane, the stream of the air is oscillate and hasn't got the straight direction though the inner tube. After the inlet it is immediately bended to the vicinity of the wall. The movement of the air in the annular part of the body after the outlet tube is almost immobile. By the effect of the geometry the output stream is rotating in the outlet tube. Maximal velocity at the inlet is $427 \mathrm{~m} \cdot \mathrm{s}^{-1}$ and at the outlet is $424 \mathrm{~m} \cdot \mathrm{s}^{-1}$ for the maximal operation condition of the Nosko ${ }^{\circledR}$ derived from vacuum cleaner limitation.

The results of the simple experiment investigation the streamlines of the trace in the body is presented in the Figure 6 . This streamlines partially agree with those obtained by simulation. The bending of the input stream can be observed in the both results. The white region in the left of the Figure 6 represent generated fog which is sucked into the body by arranged under pressure. In the right side of the Figure 6 the backflow in the central tube which is generated by reflection of the part of the main stream is slightly visible (left top near the inner wall). This backflow can be observed also in the Figure 4 of velocity contours as well as in the Figure 5 of stream lines.

In the Figure 7, the kinetic energy of turbulence contour is presented. The highest value of the kinetic energy of turbulence occurs next to the inlet region, where mostly by expansion of the stream the central vortex in the velocity filed is created. This central vortex push out the streamlines of the flux near to the wall. This vortex passes through central inner tube and it breaks down by viscous forces into the small vortices and it continuously dissipates into the heat. High intensity of the turbulence also occurs at the outlet of the body, which is expected due to flow contraction and complicated fluid flow from annular cross section into the small outlet tube.

The described and investigated complicated flow condition of the air affected by the body design of manufacturer serves primarily for phlegm separation from the air. This body design ensures that the phlegm remains in the body and eliminates the suction of the phlegm into the tube of the vacuum cleaner. 

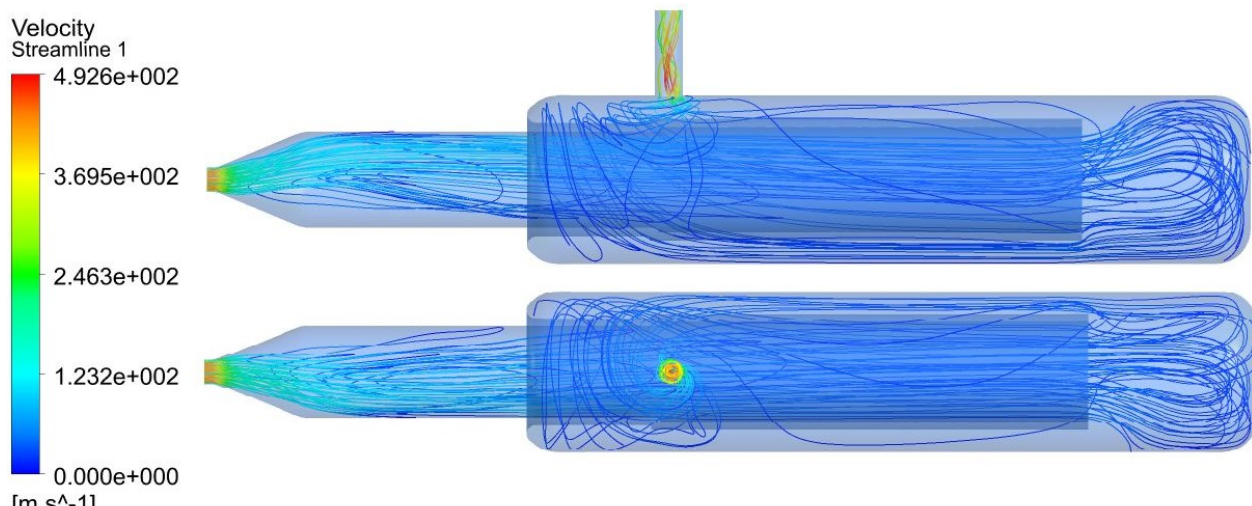

$\left[\mathrm{m} \mathrm{s}^{\wedge}-1\right]$
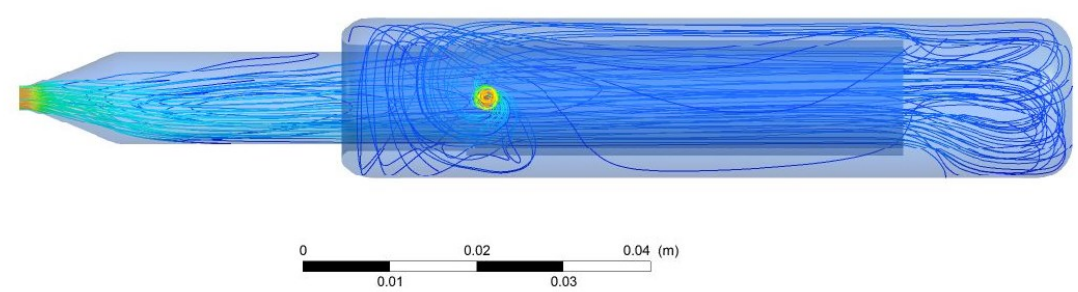

Fig. 5. Streamlines for 2.6 litres. $\mathrm{s}^{-1}$.

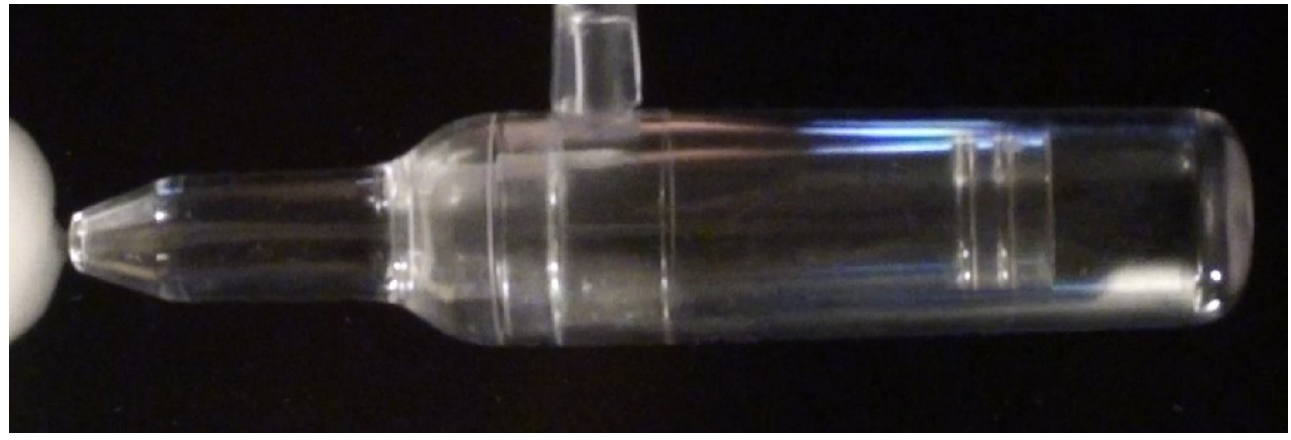

Fig. 6. Record of the tracer streamline obtained by experiment.

Turbulence Kinetic Energy

Contour 1
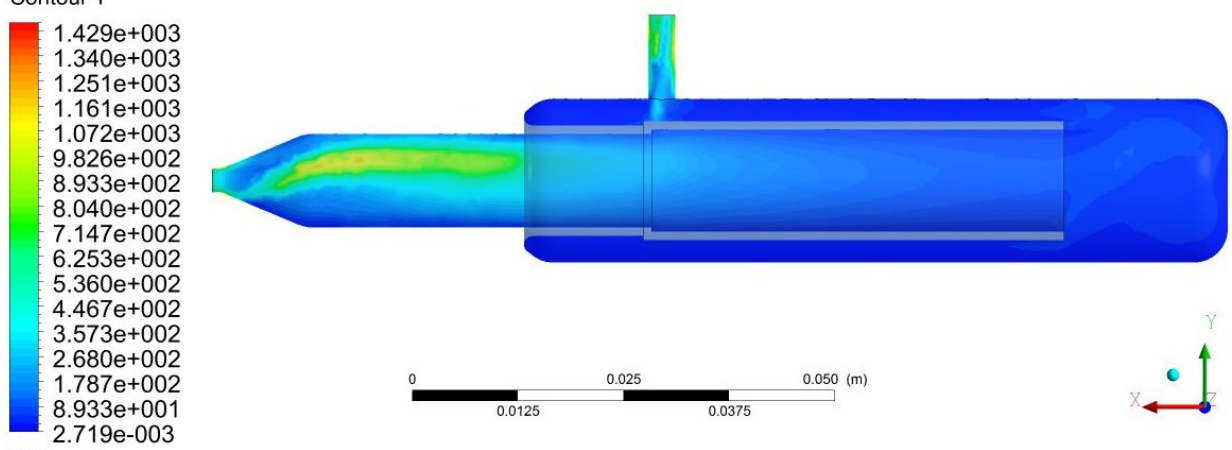

[J kg^-1]

Fig. 7. Kinetic energy of turbulence flow contours for 2.6 litres. $\mathrm{s}^{-1}$. 


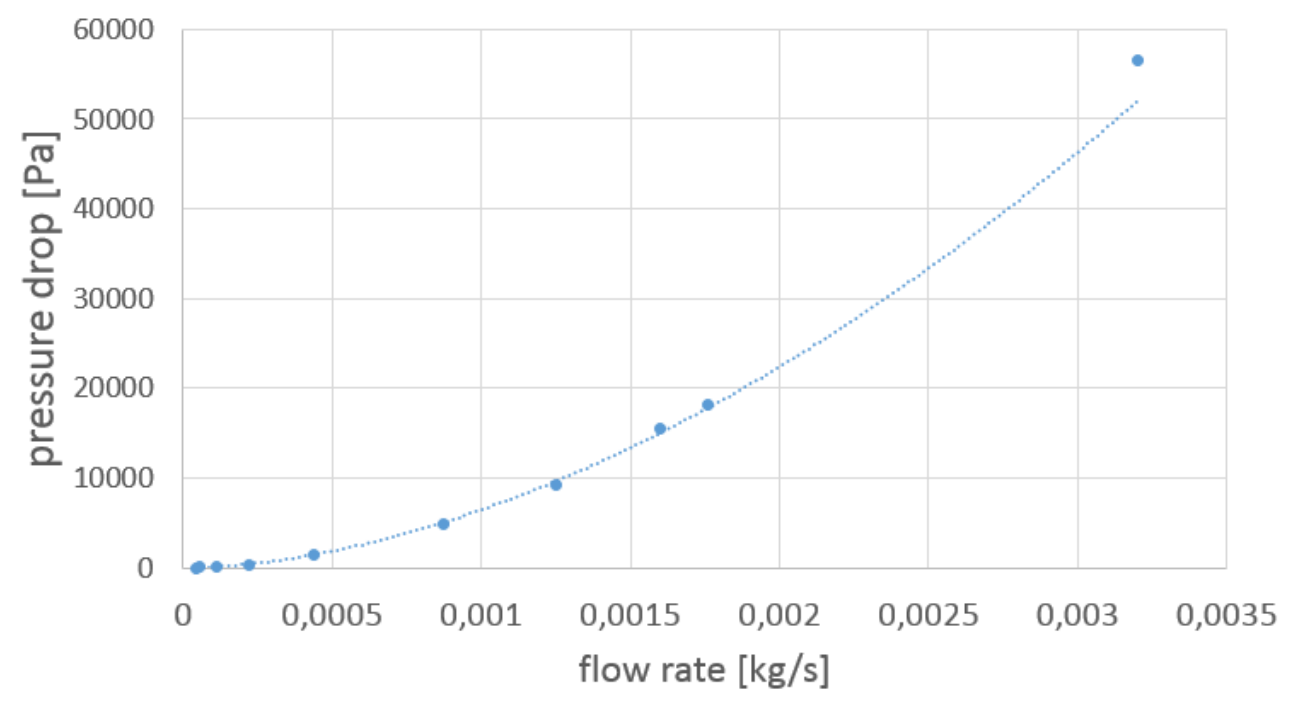

Fig. 8. Flow characteristic of the Nosko ${ }^{\circledR}$, points represent particular simulation results and line is presentation of the equation (1).

The CFD model of the Nosko ${ }^{\circledR}$ was utilized for assessment of flow characteristic, see Figure 8. Several flowrates were investigated to obtain related value of pressure drop. By regression analysis the final curve of the flow characteristic or local pressure drop calculation was obtained, and it mathematic formula is presented by equation (1)

$$
\Delta p=a \cdot m^{b}
$$

where $a=2.9 \times 10^{9} \mathrm{~Pa} .\left(\mathrm{kg} \text {. } \mathrm{s}^{-1}\right)^{-\mathrm{b}}$ with $95 \%$ confidence bounds (2.6 to 3.2$) \times 10^{9}$ and exponent $b=1.89$ with $98 \%$ confidence bounds (1.87 to 1.91). Root Mean Square Error is $\mathrm{RMSE}=122.9$ and coefficient of determination $R^{2}=1$.

Maximal flow rate for the pressure drop $30 \mathrm{kPa}$ is 2.6 litres. $\mathrm{s}^{-1}$ and mean velocities in the inlet is $427 \mathrm{~m} . \mathrm{s}^{-1}$ and outlet is $353 \mathrm{~m} \cdot \mathrm{s}^{-1}$. However, these results look extreme and can be considered as harmful condition they have to be interpreted carefully. These values are extremely influenced by phlegm presence, pressure drop of the sinus nasal of individual person as well as the operation parameters set up of the vacuum cleaner. These values can be reached by condition before connection to the human nose and the hydraulic resistance of the sinus nasal can significantly reduce the value of the flow rate. With knowledge of working curve (pneumatic characteristic) of the vacuum cleaner together with hydraulic resistance of the sinus nasal and obtained hydraulic characteristic of the Nosko ${ }^{\circledR}$ by this article, the working point of the connected system can be achieved. It is evident that real values of working point will be then considerably lower. On the other hand, the value of the air flow rate is not so far from the values presented by article [11] for adult human breathing.

\section{Conclusion}

The article deals with investigation of the pressure and velocity conditions in selected home health care accessory for babies. The objective was to find out the maximal under-pressure and air velocity, which is possible to reach by accessory Nosko ${ }^{\circledR}$ using standard home vacuum cleaner recommended by manufacturer of the accessory. CFD software was used 
for prediction of the flow characteristics of the air in the body of the accessory. Also simple experiment with the trace was performed with the Nosko ${ }^{\circledR}$ to observe the streamline inside the transparent body. Standard techniques of CFD tool were used. The obtained results were compared and the agreement between simulation and experiment is satisfactory. The assessed flow behaviour of the air predicted by CFD tool was utilized for explanation of the main function of the accessory. Also the flow characteristic of the Nosko® was obtained by systematic simulation and can be applied for further engineering calculation and investigation of the system human nose - Nosko ${ }^{\circledR}$ - home vacuum cleaner. Maximum flow rate of the air through the accessory under maximal under pressure generated by home vacuum cleaner was 2.6 litres. $\mathrm{s}^{-1}$ which is comparable with results of investigation of the human breathing presented by [11].

This work was supported by SGA (Student Grant Agency) Jan Evangelista Purkyně University in Ústí nad Labem, Czech Republic (UJEP-SGS-2017-48-003-3).

\section{References}

1. R.P. Benedict, Fundamentals of pipe flow (John Wiley \& Sons, New York, 1980)

2. R.W. Miller, Flow Measurement Engineering Handbook (Osborne-McGraw-Hill, New York, 1983)

3. U.G. Pirumov, G.S. Roslykov, Gas flow in Nozzles (Springer-Verlag Berlin Heidelberg, New York, 1986)

4. Y. Yu. M. Shademan, R. M. Barron, R. Balachandar, Eng. App. Comp. Fluid Mechanics, 6(3), 412-425 (2012)

5. H. Koskela, Energy and Buildings, 30, 1011-102 (2004)

6. R. Lenhard, T. Puchor, EPJ Web of Conferences, 143 (2017)

7. C. Badock, R. Wirth, A. Fath, A. Leipertz, Intl. J Heat and Fluid Flow 20, 538-544 (1999)

8. K. Petera, M. Dostal, M. Věř́ššová, T. Jirout, Chem. Biochem. Eng. Q., 31(3), 343-352 (2017)

9. I.B. Celik, U. Ghia, P.J. Roache, Ch. J. Freitas, J. Fluids Eng 130(7), (2008)

10. IEC 312 / DS/EN 60312 Standard, Vacuum Cleaners for Use in the Household Methods for Measuring the Utility Properties

11. J. Pertuze, A. Watson, N.B. Pride, J. Appl Physiol, 70(3), 1369-76 (1991) 